\section{Astrocytes in the air}

In recent years it has become increasingly apparent that astrocytes have roles beyond supporting neurons. Now, Gourine et al. add to these cells' repertoire by showing that astrocytes in the brainstem contribute to respiratory control.

Because astrocytes provide a link between neurons and the vasculature, the authors investigated the possibility that they integrate levels of $\mathrm{CO}_{2}$ and $\mathrm{pH}$ in the blood and the brain parenchyma, and relay this information to chemosensitive neurons in brainstem areas that control breathing. They measured the reactivity of astrocytes near the ventral surface of the medulla (VSM) to changes in $\mathrm{pH}$ by monitoring intracellular $\mathrm{Ca}^{2+}$ concentrations. Experiments in rat brainstem slices showed that a 0.2 unit decrease in $\mathrm{pH}$ induced an immediate increase in $\mathrm{Ca}^{2+}$ levels in VSM astrocytes. Astrocytes located adjacent to the VSM vasculature were particularly sensitive to changes in $\mathrm{pH}$. The cellular $\mathrm{Ca}^{2+}$ response to acidification is propagated across astrocytic networks through the release of adenosine triphosphate (ATP). In brainstem slices, a 0.2 unit $\mathrm{pH}$ decrease triggered ATP release in the VSM, and this effect was prevented by blocking ATP signalling.

How are these astrocytic responses to changes in $\mathrm{pH}$ linked to the control of breathing? Neurons in the retrotrapezoid nucleus (RTN), which lies adjacent to the VSM, are known to be chemoresponsive and are thought to have a role in the central control of respiration. The authors showed that a $\mathrm{pH}$ decrease induced depolarization and intracellular $\mathrm{Ca}^{2+}$ elevations in RTN neurons. These responses were abolished by blocking ATP signalling, indicating that they are mediated by ATP. To further confirm the link between astrocyte activation and RTN neuron activation, the authors used optogenetic tools to selectively trigger $\mathrm{Ca}^{2+}$ responses in VSM astrocytes in brainstem slices. This triggered a profound ATP release and ATPdependent depolarization of RTN neurons. Moreover, optogenetic activation of VSM astrocytes in anaesthetized, vagotomized, artificially ventilated rats increased respiratory activity, demonstrated by an increased discharge of the phrenic nerve (which innervates the diaphragm).

This study shows an unexpected role for astrocytes in the operation of an important homeostatic reflex that is essential for breathing - a role that was assumed to be performed exclusively by defined neuronal populations in the brainstem.

\section{Leonie Welberg}

ORIGINAL RESEARCH PAPER Gourine, A. V.

et al. Astrocytes control breathing through $\mathrm{pH}$-dependent release of ATP. Science $\mathbf{3 2 9}$, 571-575 (2010)

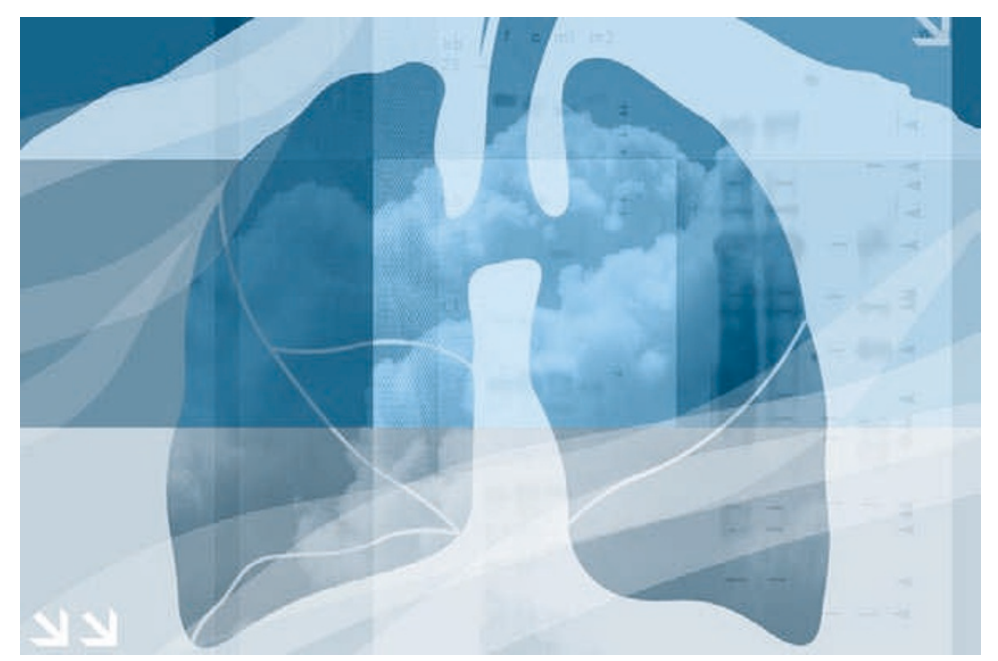

